\title{
Eban HIV/STD Risk Reduction Intervention: Conceptual Basis and Procedures
}

\author{
NIMH Multisite HIV/STD Prevention Trial for African American Couples Group
}

\begin{abstract}
Objective: To describe the Eban HIV/STD Risk Reduction Intervention being evaluated in the NIMH Multisite HIV/STD Prevention trial for heterosexual African American couples, including the integrated theoretical framework, the structure, core elements and procedures of the intervention, and how the content was shaped by culturally congruent concepts to address the needs of the study target population.
\end{abstract}

Design: The Eban HIV/STD Risk Reduction Intervention is designed to address multilevel individual-, interpersonal-, and community-level factors that contribute to HIV/STD transmission risk behaviors among heterosexual African American couples who are HIV serodiscordant.

\begin{abstract}
Methods: The Eban HIV/STD Risk Reduction Intervention employs a mixed modality, couple-based approach that is based on an integrated ecological framework incorporating social cognitive theory and uses an Afrocentric paradigm that is informed by previous evidence-based couples HIV prevention interventions. For this randomized controlled trial, African American serodiscordant couples were recruited from 4 urban sites (Atlanta, Los Angeles, New York, and Philadelphia) and were randomized to either the Eban HIV/STD Risk Reduction Intervention (treatment condition) or a Health Promotion Intervention that served as an attentional control condition. Both interventions had 4 individual couple sessions and 4 group sessions, but only the treatment condition was focused on reducing HIV/STD risk behaviors. Behavioral and biological data were collected at baseline, immediately after the intervention, and at 6 and 12 months. The theoretical framework, core elements, and content of each session are described and lessons learned from this intervention trial are discussed.
\end{abstract}

Results: An HIV prevention intervention combining couple and group sessions can be feasibly implemented with African American HIV-serodiscordant couples who remain at high risk of HIV/STD transmission. The lessons learned from the trial suggest that the participants responded very well to both the couple and the group sessions. Participant feedback suggests that the cultural congruence of the intervention and use of African American cofacilitators made them feel comfortable disclosing risky behaviors. Participant

Received for publication June 18, 2008; accepted June 20, 2008.

From the Center for Mental Health Research on AIDS, National Institutes of Health, Bethesda, MD.

Correspondence to: NIMH Multisite HIV/STD Prevention Trial for African American Couples, Center for Mental Health Research on AIDS, Division of AIDS and Health and Behavior Research, National Institutes of Mental Health, National Istitutes of Health, 6001 Executive Boulevard, Room 6219B, Bethesda, MD 20892 (e-mail: wpequegn@mail.nih.gov).

Copyright (C) 2008 by Lippincott Williams \& Wilkins feedback also suggests that the intervention's couple-based focus on enhancing dyadic communication and decision-making skills was key to helping the couples work together to overcome barriers to using condoms.

Conclusions: Participant and facilitator evaluations of the Eban Risk Reduction Intervention suggest that couples responded well to the Afrocentric content and mixed modalities of the intervention sessions. Couple sessions were optimal for enhancing interpersonal and microlevel factors, including communication, problem solving, and decision making.

Key Words: African American, behavioral intervention, couplelevel, culturally congruent, HIV, sexual behavior

(J Acquir Immune Defic Syndr 2008;49:S15-S27)

\section{INTRODUCTION}

The NIMH Multisite HIV/STD Prevention Trial for African American Couples (hereafter trial) is a 2-arm randomized controlled trial (RCT) evaluating the impact of the Eban HIV/STD Risk Reduction Intervention, a culturally congruent, couple-based intervention to reduce HIV/STD transmission risks among African American serodiscordant couples. This multisite RCT was implemented in 4 metropolitan areas in the United States: Atlanta, Los Angeles, New York, and Philadelphia. A wide range of venues of recruitment was employed to identify and enroll participants for this trial, including street outreach, media, churches, community-based organizations, and hospital-based clinics. The trial's primary study aim was to estimate the effects of the Eban HIV/STD Risk Reduction Intervention on 2 primary end points: biologically confirmed sexually transmitted disease (STD) incidence and the percentage of self-reported protected sexual acts of condom use with study partners.

The purpose of this article is to describe the Eban HIV/STD Risk Reduction Intervention employed in this trial, including the rationale and empirical basis that informed the design of the intervention, the underlying theoretical framework, the structure and core elements of the intervention, the content of each session, and the lessons learned from the trial.

\section{RATIONALE AND EMPIRICAL BASIS FOR EBAN HIV/STD RISK REDUCTION INTERVENTION}

Among diseases that disproportionately affect African Americans, the HIV/AIDS epidemic has had a particularly devastating effect across the United States. In 2004, HIV/AIDS was among the 3 leading causes of death for African American men aged 25-54 years, among the 4 leading 
causes of death for African American women aged 20-54 years, and the leading cause of death among African American women 25-34 years of age. ${ }^{1}$ Heterosexual contact is the most frequent route of transmission among African American women and the second leading conduit among African American men. ${ }^{2}$ The magnitude and continued heterosexual spread of HIV among African Americans suggests a scientific, moral, and ethical imperative to develop and disseminate effective prevention interventions that may address the specific issues and risk behaviors that African American HIVserodiscordant couples present.

A meta-analysis of HIV prevention interventions found that most prevention interventions were consistently less effective for African Americans, ${ }^{3}$ highlighting the need for culturally congruent approaches. A few recent RCTs have tested culturally congruent HIV prevention interventions for African Americans, mostly based on social cognitive principles and an empowerment-based approach. ${ }^{4-7}$ These culturally congruent interventions have been found to be efficacious in increasing condom use, reducing risk behaviors, and/or decreasing STDs among African Americans. ${ }^{4-8}$ In their meta-analysis of the effectiveness of HIV interventions, Logan et $\mathrm{al}^{9}$ also found that interventions, including modules focusing on culture and race, had larger weighted mean effect sizes than those without, further highlighting the efficacy of culturally congruent interventions.

Although most HIV/STD risk reduction interventions have been conducted at the individual level, a couple-based approach may be more efficacious and consistent with cultural values of African Americans. In addition, accumulating research suggests that couple-based HIV prevention interventions may be more efficacious in promoting condom use among HIV-serodiscordant couples than traditional HIV prevention interventions aimed at individuals or groups. ${ }^{10-15}$ The earliest evidence suggesting the efficacy of couple-based HIV prevention interventions comes from voluntary counseling and HIV testing (VCT) studies conducted abroad. A recent review of these studies found that VCT provided to couples was more effective at increasing condom use than individual counseling and testing. ${ }^{716-21}$ Deschamps et $\mathrm{al}^{13}$ examined whether couple counseling would reduce sexual risk behavior among 148 couples. Before counseling, only $9 \%$ of the couples used condoms; at follow-up, $40 \%$ used them. Allen et $\mathrm{al}^{17}$ conducted a study of VCT among 1458 Rwandan women. At the 2-year follow-up, HIV-negative women whose partners had participated were $50 \%$ less likely to seroconvert than were those whose partners had not participated. In one of the first studies to evaluate a couple-based VCT in the United Status, Padian et $\mathrm{al}^{12}$ examined behavior change among 144 HIV-serodiscordant heterosexual couples who received intensive couple counseling. About half $(49 \%)$ of the couples reported consistent condom use before the intervention, whereas $88 \%$ reported consistent condom use at first followup, but the increase in condom use was not maintained at subsequent follow-ups. In the first multicountry RCT to test the efficacy of VCT with couples, the Voluntary HIV-1 Counseling and Testing Efficacy Study Group ${ }^{15}$ randomly assigned 586 couples to receive either a couple-based VCT or a basic health information intervention. Couples assigned to the VCT condition were more likely to report decreasing unprotected intercourse.

El-Bassel et $\mathrm{al}^{22,23}$ conducted one of the first RCTs to test the efficacy of a relationship-based HIV/STD prevention intervention with low-income urban couples in the United States. For this study, 217 couples at elevated risk of HIV/ STDs were randomized to 1 of 3 conditions: (1) a 6-session relationship-based HIV/STD prevention intervention provided to couples; (2) the same 6 sessions provided to the women alone; or (3) a 1-session HIV/STD information session provided to the women alone, which served as the control condition. El-Bassel et al. ${ }^{22,23}$ found that the HIV prevention interventions provided to the couples or women alone were efficacious in reducing unprotected sex at both the 3-month and the 12-month follow-up assessments compared with the informational control condition. The results of this study supply additional evidence of the sustained efficacy of a relationship-based intervention for increasing condom use among low-income urban couples.

Although these cumulative findings suggest the efficacy of couple-based HIV prevention interventions with different populations, most of these studies remain limited by one or more methodological drawbacks, including relatively small samples, lack of a randomized control design, lack of biologically confirmed outcomes, and lack of an attentional control group. Nevertheless, the literature suggests that a couple-based HIV prevention intervention with African American HIV-serodiscordant heterosexual couples using a mixed modality of individual couple sessions and couple group sessions may be efficacious for several reasons.

Couple-based approaches have been found to be effective in causing positive change in (a) increasing commitment in a relationship to protecting each other from HIV/STDs; (b) reducing gender power imbalances that influence condom use; and (c) increasing sexual communication and negotiation skills. ${ }^{4,24-32}$

Couple groups are especially effective in causing positive change in (a) building social support and peer norms to protect themselves and their partners from HIV/STDs; (b) reducing HIV-associated stigma as a barrier to prevention; and (c) increasing cultural pride and collective self-efficacy to protect their community from HIV/AIDS. Group discussions with HIV-serodiscordant couples have helped to reduce HIV risk. $^{33}$ Intercouple interactions allow intimate partners to empathize with each other, share feelings about the influence of HIV/AIDS on family life, and decrease isolation. Behavior change may be prompted by identification with other couples "who have been there." ${ }^{34}$

\section{THEORETICAL FRAMEWORK OF EBAN RISK REDUCTION INTERVENTION: OVERVIEW}

The theoretical framework for the Eban HIV/STD Risk Reduction Intervention integrates components of social cognitive theory $(\mathrm{SCT})^{35}$ and an Afrocentric paradigm ${ }^{36}$ into a relationship-oriented ecological framework ${ }^{37}$ that addresses multilevel risk and protective factors (see Fig. 1) associated with HIV/STD risk reduction among African American HIVserodiscordant couples. SCT informed the factors in the 


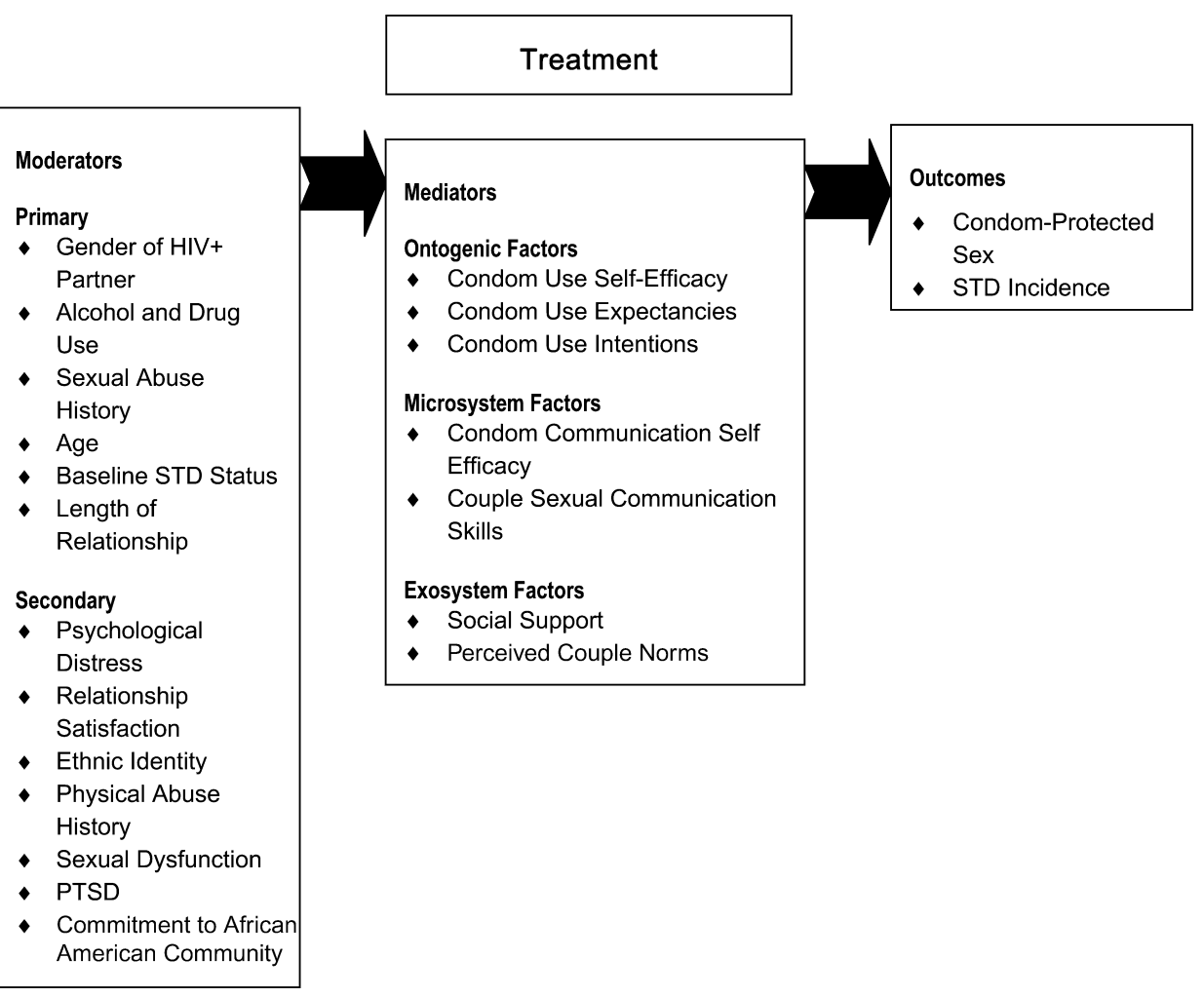

FIGURE 1. Conceptual framework for Eban HIV/STD risk reduction intervention. efforts to integrate Bronfenbrenner's ${ }^{37}$ multilevel analysis of the social context and individual development. The heuristic value of this integrated ecological perspective derives from the recognition of the broad range of distal and proximal factors influencing human development and behavior. ${ }^{37}$ This model allows for interpretation of where any particular factor fits most appropriately within the multilevel framework. The goal of this multilevel integrated theoretical framework is to characterize the relative "weights" assigned to each multilevel determinant of HIV risk behaviors. This framework organizes key factors facilitating or preventing HIV risks into 4 different levels (individual-, interpersonal-, community-, and macrolevel factors), which in turn are influenced by background variables as described below. These multilevel factors interact with each other and may change over time, leading to shifts in HIV risk behaviors among African American HIVserodiscordant couples.

These multilevel factors may include an individual's background, the dyadic context in which members of a couple relate to each other, the immediate social context, and the broader cultural values and beliefs that shape the relationship context in which HIV risk behaviors occur. "The ontogenetic level" refers to the personal factors that are associated with an individual's developmental history and the individual-level factors associated with HIV behavioral change, which are detailed in the SCT of behavior change. "Microlevel" factors are part of the immediate intimate relationship context in which sexual activity and HIV risk and protective behaviors take place, including sexual communication and negotiation skills, relationship dependencies, and balance of sexual decision-making power. Recent research underscores the 
importance of these dyadic factors in understanding why couples engage in unprotected sex. "The exosystem level" refers to all social factors that impinge upon the immediate setting by acting as external stressors or buffers on the likelihood of engaging in risky behavior. These include socioeconomic status, peer norms about safer sex, and access to social support. Several studies have tied peer norms about condom use to sexual risk behavior. ${ }^{39,40}$ Heterosexual relationships particularly in low-income urban communities tend to be embedded in traditional gender roles where men have greater power in making decisions about sexual and reproductive issues. $^{41-43}$

As shown in the above figure, this ecological framework integrates components from SCT and the Afrocentric paradigm in the selection of multilevel risk and protective factors that are posited to influence HIV/STD risk reduction behaviors among African American HIV-serodiscordant couples.

\section{SOCIAL COGNITIVE THEORY}

SCT has guided many behavioral HIV prevention studies. $^{21,22,44,45}$ Most HIV risk behavior models that are guided by SCT focus on mechanisms that predict risk and protective behaviors and provide guidance on how to change behaviors. Recent research has acknowledged the dyadic, interactional nature of HIV risk behavior and has expanded the focus of SCT to couple- or community-level mediating factors associated with HIV risk reduction. ${ }^{46,47}$ The tenets of SCT used in this study were self-efficacy, outcome expectancies, and behavioral skills. Self-efficacy is the belief in one's ability to implement behaviors required to produce desired outcomes. It is also described as individual technical competence in condom use skills. Whether people consider changing their behavior, the degree of effort they invest in changing, and the long-term maintenance of their behavior change is determined by self-efficacy. ${ }^{35,48,49}$ Further, self-efficacy is a strong predictor of condom use. ${ }^{48,49}$ Because this study's dyadic focus is on promoting HIV/STD prevention among couples, selfefficacy is conceptualized primarily as couple efficacy in communicating and negotiating using condoms. ${ }^{22}$

Outcome expectancies refer to the individual's perceived benefits and costs of performing a certain behavior. Key outcome expectancies for this study were beliefs that using condoms would be an effective means of preventing HIV/STDs (benefits) and that condom use would not lead to negative partner reactions or diminish trust and sexual intimacy (costs).

The behavioral skills concept in SCT is concerned with the person's ability to successfully execute a given behavior. One of the major contributions of SCT has been to offer strategies to teach skills to change behaviors. There were 2 major relevant behavior change strategies: (1) guided practice with performance accomplishments that provide rewards, increasing skills, and self-efficacy and (2) observational learning or vicarious experience by observing other people performing the skill and receiving reinforcement. Both strategies build skills and self-efficacy that may better enable couples to work through common risk situations.

\section{AFROCENTRIC PARADIGM}

Cultural factors can filter and shape the reasons that people engage in certain behaviors. ${ }^{50}$ Culture encompasses a group's history, values, knowledge, and behavioral norms that are explicit or implicit in social interactions. ${ }^{51,52}$ A variety of African American cultural beliefs are also influenced by health disparities, poverty, and history of perceived racism and oppression experienced in the United States, ${ }^{52}$ yet these factors are rarely incorporated in HIV risk reduction interventions, especially for heterosexual African American couples. Consequently, a more ethnic-specific approach was used in the Eban interventions.

An Afrocentric paradigm provided a more comprehensive inclusion of individual, interpersonal, cultural, and societal factors valued within diverse groups of African American people who are overlooked in traditional HIV interventions. The organizing principles include spirituality, harmony, collective responsibility, oral traditions, sensitivity to emotional cues, authenticity, concurrent time orientation to past, present, and future, and group affiliation. ${ }^{53,54}$ The central assumption is that enhancement of Afrocentric values and ethnic identity will increase resiliency and the motivation to work through barriers to HIV risk reduction. ${ }^{53}$

The importance of spirituality and religious involvement in health beliefs among African Americans has been well documented by research. ${ }^{55}$ The Afrocentric paradigm acknowledges the importance of spiritual beliefs, while emphasizing self-protection, and the value of relationships, as well. These factors seem contradictory but are consistent with cultural, spiritual, and Afrocentric thought. ${ }^{56}$ The focus on self-protection is also endorsed by these values. The use of language and perspectives that are based on religion, culture, and Afrocentrism is more familiar to African Americans than public health-oriented risk reduction messages. The realities of life as an African American are powerfully expressed by vocal traditions, people affirming each other's thoughts, feelings, circumstances, or experiences in group discussions. ${ }^{53}$ This paradigm also endorses the use of Ebonics (natural speech patterns of African Americans) to establish a conversational tone to ensure that participants perceive discussion topics in a "real-life" context. This down-to-earth approach to group discussions increases participation in and completion of the intervention. ${ }^{53}$ Traditional and contemporary African American literary and popular cultural references were also used to further enhance and integrate cultural specificity and pride about protecting African American communities from HIV/STDs. The Eban intervention also addressed socioeconomic contexts associated with HIV risks, including poverty, disenfranchisement from health care systems, and perceived racism. Finally, an Afrocentric approach emphasized an equitable relationship between group facilitators and participants with open, respectful, and nonconfrontational or nonjudgmental communication. In the Eban intervention, the 7 principles of Nguzo Saba (unity, self-determination, collective work and responsibility, cooperative economics, purpose, creativity, and faith) that guide the Afrocentric worldview and belief systems of native groups of Africa ${ }^{36}$ informed the content of the intervention and the style of its 
delivery. Nguzo Saba brings couples, families, and communities together, promotes respect for traditions, and highlights the sociopolitical and racial realities faced by Africandescended people affected by HIV (see Table 1). Consistent with the Afrocentric paradigm, the 7 principles promote individualism in skill development and collectivism in relationship building and behavior change. ${ }^{36,57}$

\section{USE OF THE COMMUNITY ADVISORY BOARD}

Effective interventions frequently involve communitybased participatory approaches throughout the design and implementation of clinical trials. A Community Advisory Board (CAB) was established to ensure that the Eban HIV/ STD Risk Reduction Intervention was culturally congruent and tailored to the needs, issues, and worldviews of many urban African American HIV-serodiscordant couples. CAB feedback improved the quality and contextual relevance of the intervention and facilitator training protocols, established the study's visibility in black communities, and served as an active and influential bridge between the research team and these communities. [For a discussion of the role of the $\mathrm{CAB}$, see The Role of Community Advisory Boards (CABs) in Project Eban in this issue.]

\section{STRUCTURE AND DELIVERY OF THE INTERVENTION}

The integrated theoretical framework guiding this study is incorporated into the structure, format, and content of the curriculum. The mixed modality structure approach to the intervention allowed for the personal, interpersonal, community, and societal levels to be addressed. The intervention consists of 8 weekly 2 -hour sessions that were delivered during the day, evening, or weekend, depending on the couple's availability. The intervention is highly structured and implemented by male and female African American cofacilitator pairs who were trained to adhere to the Eban HIV/STD Risk Reduction Intervention Manual. The cofacilitators introduced and modeled couple communications, negotiations, and decision-making skills to increase condom use and reduce risk behaviors. The intervention format relies on dyadic and group processes and allows facilitators to employ intervention strategies that address factors influencing risk behavior among HIV-affected couples.

For example, the intervention is delivered in individual couple sessions and 4 couple group sessions. Individual couple sessions were optimal to address interpersonal or microlevel factors associated with sexual risk reduction, including communication, problem solving, and decision making. Sessions with groups of couples were designed to address community-level factors associated with safer sex. These factors include (1) increasing positive peer norms for condom use and safer sex by emphasizing the threat of HIV to African American communities, (2) destigmatizing life as an African American couple affected by HIV, and (3) increasing social support and coping strategies for HIV risk reduction.

In the first half of session 1, a group of 3-5 couples met with their cofacilitator team; in the second half, participants met in single-gender groups with the same-gender facilitator. In sessions 2-4 and 8, each couple met separately with their cofacilitators. In sessions 5-7, the couples attended group sessions with the other couples and their cofacilitators. In session 8 , each couple met separately with their cofacilitators. Skills taught in the couple sessions were reinforced in the group sessions.

The structure of sessions 2-8 followed a general format of (1) reviewing highlights of the previous session; (2) reviewing homework including identifying difficult parts of the assignment; (3) presenting and discussing a sequence of topics on HIV/STD transmission risks, prevention strategies, and/or risk reduction skills; (4) modeling and role-playing risk reduction skills that may be used in common risky situations; (5) revisiting progress on couples risk reduction goals, identifying barriers to achieving and resetting goals for the upcoming week; and (6) reviewing homework. Facilitators were trained to help the couples identify specific barriers to using condoms consistently or implementing other risk reduction strategies (eg, getting tested for STDs). The facilitators often set up role-playing to dramatize alternative ways to handle risky situations that couples encounter and exercises to practice new concepts introduced in each session. Participants were compensated for their participation at the end of each session.

\section{CONTENT OF THE HIV/STD INTERVENTION}

The intervention components build on each other to strengthen the couple's relationship. The intervention incorporated Eban, a traditional concept of the Yoruba people of Africa, which means fence - a symbol of safety, security, and love within one's family and relationships. Group sessions promoted the concept of a village that is a community protected by the fence (Eban). Group cohesion was encouraged through a variety of group activities, including opening and closing rituals, the Talking Circle, and the setting of group rules for safety and respect. The principles of Nguzo Saba were woven into the theme and content of the sessions and used to motivate couples to use condoms consistently to protect each other and their community. Each session highlighted 1-3 principles of Nguzo Saba and incorporated them into the activities and discussions in the sessions. Of the 7 principles, 5 (ie, unity, self-determination, collective work and responsibility, purpose, and creativity) were discussed in 2 or more sessions, whereas 2 principles regarding cooperative economics were highlighted in sessions 1 and 7. Multiple elements of the intervention served to build each principle of Nguzo Saba between partners and within groups of couples.

\section{Session 1: Preparing for the Journey (Group Session)}

The objectives of the first session were to orient the couples to the purpose of Project Eban, increase their comfort with and commitment to the study, enhance cultural and gender pride, and provide information about STDs, HIV, and safer sex practices. Facilitators introduced the purpose of Project Eban. They emphasized how HIV/AIDS and STDs are affecting the black community and highlighted the importance 


\begin{tabular}{lll}
\hline TABLE 1. Seven Principles of Nguzo Saba & \multicolumn{1}{c}{ Construct } & \\
\hline Swahili & Unity & Definition
\end{tabular}

of couples working together to be safe. In the Talking Circle, participants sat in a circle holding an ankh and introduced themselves. After sharing their reasons for participating, they passed the ankh to the next person. The facilitators led a discussion on the meaning of this symbol. The group rules for participation were generated by participants and facilitators and were posted at each session. Each participant was asked to sign a contract of personal commitment and confidentiality. After orienting the participants, facilitators introduced the 7 principles of Nguzo Saba and highlighted how they could be used as a source of cultural strength and a blueprint for healthy living and positive well-being. The couples discussed what the principles meant to them and related the principles to their desire to live a healthy life and protect each other and their communities from HIV/STD transmission.

The second half of this session elicited common genderbased challenges to condom use and risk reduction for women and men and focused on enhancing the participants' comfort with both group and couple sessions. The single-gender groups discussed and identified participants' reasons for enrolling in the study. This discussion also focused on possible barriers to attendance and participation. During this discussion, facilitators explored how participants felt about mixedgender group discussions in front of their partner. Facilitators led activities and discussions designed to enhance culturally specific gender pride, focusing on the unique resilience of African American women or men as a source of motivation for HIV/STD risk reduction. The facilitators reviewed STD and HIV facts in an interactive exercise. Participants discussed a general and personal list of obstacles to condom use. The single-gender groups brainstormed strategies for overcoming these obstacles before the facilitators reconvened the couples as a village (group). Participants were given a workbook with intervention handouts and facilitators reviewed its contents and discussed the homework assignment for the next session. Participants identified what they loved about their partner and their desire to protect each other from HIV/STDs. This "Cherish Activity" was designed to enhance the couple's motivation for risk reduction.

\section{Session 2: Enhancing Couple Communication (Couple Session)}

This session integrated 3 Nguzo Saba principles: "unity," "purpose," and "collective work and responsibility." The focus was on maintaining unity in the family, community, nation, and race, preserving the legacy of ancestors, and discovering each participant's value to the community. Through the goal setting in this session, couples defined their "purpose" as a couple and examined how they can apply the principles of Nguzo Saba to their lives.

This session opened by welcoming the couple followed by a brief review of the major topics from prior sessions. The homework assignment from the prior session (Cherish Activity) was reviewed and discussed. Couples were reinforced for their progress on homework assignments, use of Nguzo Saba principles, and discussion about barriers to homework completion. This session introduced 2 core skills to be integrated in the remaining sessions (see Table 2).

The first exercise in this session was designed to help couples to recognize common but ineffective patterns of communication. A more effective communication technique, called Talk and Listen, was introduced and demonstrated with a 7-minute video clip. Talk and Listen is a core communication skill that is designed to increase listening capacity and to slow down and clarify the process of couple communication. Training couples to provide clear communication and feedback to each other grew out of sex therapy techniques. Markman et $\mathrm{al}^{58}$ used the technique in efficacious couplebased HIV prevention interventions ${ }^{23}$ and adapted it for the speaking patterns of African American couples. Using Talk and Listen, partners gain a deeper understanding of each other's viewpoints and feelings about issues of potential conflict. Partners alternate being in the role of talker or listener until they have fully heard each other's viewpoint, concerns, and feelings about a particular issue. The talking partner is asked to hold the ankh or any other meaningful symbol while talking, to be concise in communicating, and to use "I" statements when expressing concerns or feelings related to the issue. When the talker is finished, he or she passes the ankh to 
TABLE 2. Risk Reduction Curriculum Outline

\begin{tabular}{|c|c|c|c|}
\hline Session No & Type & Title & Components \\
\hline 1. & Group session & Preparing for the journey & $\begin{array}{l}\text { Couples group } \\
\text { Introducing the journey } \\
\text { Introducing the talking circle } \\
\text { Introducing the Eban symbol } \\
\text { Group rules for participation } \\
\text { Itinerary for the journey } \\
\text { Introducing the } 7 \text { principles } \\
\text { Single-gender group } \\
\text { Reviewing the purpose of the journey } \\
\text { Enhancing gender pride } \\
\text { STD and HIV facts } \\
\text { Barriers to risk reduction } \\
\text { Homework }\end{array}$ \\
\hline 2. & Couple session & $\begin{array}{l}\text { Enhancing couple } \\
\text { communication }\end{array}$ & $\begin{array}{l}\text { Opening and welcome* } \\
\text { Review last session* } \\
\text { Overview of current session* } \\
\text { Review homework* } \\
\text { Patterns of communication } \\
\text { Talk and Listen technique } \\
\text { Problem-solving skills } \\
\text { Risk behaviors and barriers to risk reduction } \\
\text { Couple goal setting } \\
\text { Homework }\end{array}$ \\
\hline 3. & Couple session & Tools for the journey & $\begin{array}{l}\text { Male and female anatomy } \\
\text { Using barrier methods correctly } \\
\text { Making safer sex fun } \\
\text { Homework }\end{array}$ \\
\hline 4. & Couple session & Sharing the load & $\begin{array}{l}\text { Identifying triggers to unsafe sex and } \\
\text { barriers for safer sex } \\
\text { Things that affect decision making } \\
\text { Working together to make decisions } \\
\text { Working together to improve our health } \\
\text { Working together to make better sexual decisions } \\
\text { Moving back to the village } \\
\text { Homework }\end{array}$ \\
\hline 6. & Group session & Strengthening the village & $\begin{array}{l}\text { Sharing the reality of HIV } \\
\text { Confronting triggers } \\
\text { Homework } \\
\text { Eban closing circle ritual }\end{array}$ \\
\hline 7. & Group session & Expanding the village & $\begin{array}{l}\text { Reviewing safer sex skills } \\
\text { Self-assertiveness skills } \\
\text { Reframing your requests } \\
\text { Building your village } \\
\text { Homework } \\
\text { Saying good-bye to the village }\end{array}$ \\
\hline 8. & Couple session & Celebrating our relationship & $\begin{array}{l}\text { Review progress on couples goals } \\
\text { Relapse prevention } \\
\text { Commitment to staying safe } \\
\text { Closing ceremony }\end{array}$ \\
\hline
\end{tabular}

*These elements were present in Sessions 2-8. 


\begin{tabular}{ll}
\hline TABLE 3. FENCE \\
\hline $\mathrm{F}$ & Finding out what is going on, defining the problem or issue \\
$\mathrm{E}$ & $\begin{array}{l}\text { Exploring each partner's feelings } \\
\mathrm{N}\end{array}$ \\
& $\begin{array}{l}\text { Naming and discussing the couples' options, } \\
\text { including the pros and cons of each one }\end{array}$ \\
$\mathrm{C}$ & $\begin{array}{l}\text { Choosing the best option to solve a problem } \\
\mathrm{E}\end{array}$ \\
\hline
\end{tabular}

the other partner. The listener is asked to paraphrase the key points and feelings that the talking partner conveyed. The listener conveys his or her understanding of the talker's communication, even if there is disagreement between them. The talker and listener reverse roles until they have completed discussing the issue.

Problem-solving skills were taught through FENCE, an acronym representing cognitive skills needed for communication regarding risk reduction. Couples were encouraged to conceptualize this skill as a way of creating a FENCE of safety around themselves and the community. "F" represented finding out what is going on, which helps define the problem or issue. "E" represented exploring each partner's feelings, which facilitates understanding of the issue and provides an opportunity to validate each partner's feelings. " $\mathrm{N}$ " represented naming and discussing the couple's options. " $\mathrm{C}$ " represented choosing the best option to solve a problem, and "E" represented executing the plan and monitoring progress.

Couples practiced using FENCE and facilitators provided guidance, praise, and constructive feedback. Through repeated guided practice in the remainder of the sessions, couples developed competence in these 2 skills. They were encouraged to use Talk and Listen to ensure that they fully understood each other's viewpoint and to use FENCE to problem solve the issue together.

In the next activity, couples identified risky behaviors they engaged in as a couple and the level of HIV/STD risk for each behavior. Finally, they set 2 goals to accomplish in the next 7 weeks: one related to reducing HIV/STD risk behavior and one involved with improving their relationship.

For homework, the couples were asked to select which strategies they would use over the next week (HIV risk and relationship) using the Talk and Listen and FENCE techniques to discuss their goals (Practice Skills Learned).

\section{Session 3: Tools for the Journey (Couple Session)}

The Nguzo Saba principle of "purpose" was implemented by couples acquiring the knowledge and skills necessary to protect themselves. The concept of creativity was also integrated into this session to highlight participants' talent and imagination to build harmony and beauty in their community. The intervention also emphasized how knowledge, wisdom, and creativity have historically been emphasized in African culture and societies.

The homework (Practice Skills Learned) from the previous session was reviewed. Couples recorded their progress on goals of practicing Talk and Listen and FENCE on a Couple Goal Form and rewarded themselves for progress.
Alternative methods of managing situations that arose in the homework were discussed and practiced in role-plays.

After reviewing the homework, the cofacilitators reviewed male and female reproductive anatomy using pictures and anatomical models, described in detail how HIV/STD transmission may occur, and also discussed common sexual problems that couples experience (eg, erectile difficulties, inorgasmia, and vaginal dryness) that may influence their ability to use condoms. Couples were asked to use Talk and Listen to discuss how to improve their sex life and relationship. Cofacilitators demonstrated how to use both male and female condoms correctly. Both partners were asked to practice correct use of condoms using anatomical models. Cofacilitators reviewed which lubricants were safe to use with condoms and how to eroticize condom use. Oral sex, anal sex, and mutual masturbation were defined, and safer sex practices for each were identified (eg, use of dental dams). Finally, couples learned ways to enhance safer sexual techniques to make sex more enjoyable using the Eban Café exercise (see Table 4). The Eban Café is a menu of safer sex activities from which couples "order" to select enjoyable behaviors using communication about sexual activities and safer sex practices. Cofacilitators guided the couple in practicing how to order from the Eban Café, emphasizing the need to choose behaviors that both partners would prefer to engage in. Cofacilitators also worked with couples to review progress on their goals.

For homework, couples were asked to plan a date night and use their creativity to find exciting ways to incorporate condoms and other safer sex methods (Date Night). They were provided a bag of "condom-ments" to take home to promote safer sex behaviors when ordering from the Eban Café Menu. Couples were also referred to a handout that listed what they could do on a date for under $\$ 20$.

\section{Session 4: Sharing the Load (Couple Session)}

The Nguzo Saba principles of unity, collective work, and responsibility emphasize how the decisions couples make affect their health. The relationship between individual risk and heightened risks to the relationship, family, and larger community was discussed in this session. If one member of the couple increased the risk of their partner, there were costs and potential consequences to the children and family and the larger community.

During homework review (Date Night), couples discussed their date, including whether they used condoms or other methods to reduce sex-related risk. Any difficulties with using safer sex practices were addressed using the Talk and Listen techniques to understand each person's perspectives on an issue and then using FENCE to problem solve it.

The cofacilitators introduced the first main topic, specifically identifying the top 3 triggers (events and circumstances) that could lead to unsafe sex. Couples practiced their problem-solving skills, using FENCE, to address one of these triggers. In the next activity, the cofacilitators discussed how issues associated with the balance of power and gender roles in their relationship could influence decisions related to engaging in risky behaviors and using condoms. The curriculum highlighted how a history of childhood sexual abuse or partner violence could heighten risky sexual or drug-related 
TABLE 4. Eban Café [Before Ordering, Prepare a Bowl of "Condom-ments" (Male and Female Condoms, Water-Based Lubricants Such as KY Jelly, Massage Oils, Sex Toys That have been Properly Cleaned, Etc.)]

\begin{tabular}{|c|c|}
\hline & $\begin{array}{l}\text { Level of } \\
\text { Risk }\end{array}$ \\
\hline \multicolumn{2}{|l|}{ Appetizers/Starters } \\
\hline Foot massage & 0 \\
\hline Play with partner's hair & 0 \\
\hline Read partner poetry & 0 \\
\hline Watch sexy video with partner & 0 \\
\hline Sing partner a love song & 0 \\
\hline Dance close with partner & 0 \\
\hline Take a walk in the moonlight & 0 \\
\hline Back massage & 0 \\
\hline Describe "sexy" love scene to partner & 0 \\
\hline Listen to "sexy" music with partner & 0 \\
\hline \multicolumn{2}{|l|}{ Intermezzo/middle course } \\
\hline Give partner a full body massage (clothes on) & 0 \\
\hline Partner gives you a full body massage (clothes on) & 0 \\
\hline Trace partner's body with finger & 0 \\
\hline Undress partner-slowly & 0 \\
\hline Simultaneous massage & 0 \\
\hline Perform "strip tease" for partner & 0 \\
\hline \multicolumn{2}{|l|}{ Entree/main course } \\
\hline Full body massage (no clothes) & 0 \\
\hline Bring partner to orgasm with hand & 0 \\
\hline Bring self to orgasm & 0 \\
\hline "69" with condom or dental dam* & Low \\
\hline Parallel/mutual masturbation & 0 \\
\hline Intercourse with a male condom* & Low \\
\hline $\begin{array}{l}\text { Bring partner to orgasm with mouth using } \\
\text { condom or dental dam* }\end{array}$ & Low \\
\hline Trace partner's body with tongue & 0 to Low \\
\hline "Chef’s Special"* & 0 to ? \\
\hline \multicolumn{2}{|l|}{ Dessert } \\
\hline Intercourse with female condom* & Low \\
\hline Shower with partner & 0 \\
\hline Tell partner a fantasy & 0 \\
\hline Intercourse with condom in a position you have never tried* & Low \\
\hline Second Helping* (anything on the menu) & Low \\
\hline
\end{tabular}

0 equals no risk. You will need condom-ments for choices with a*

behaviors and depression or posttraumatic stress. Facilitators provided examples of how past experiences could affect current sexual decision making. The Talk and Listen technique along with cognitive strategies to disclose and separate abusive experiences and their subsequent psychological effects from current decisions were recommended for couples to further discuss these issues. Referrals for counseling services that specialized in abuse-related trauma and HIV risk practices were also offered. Thus, participants were not required to disclose their past experiences to partners but were provided with information to assist them in making decisions about seeking counseling to further discuss trauma-related events in their lives.
In the next activity, couples discussed other issues (eg, use of alcohol or drugs) in their relationship that may have affected their sexual risk-taking behaviors. A game called the "Wheel of Relationship Issues" was introduced, in which couples spin the wheel to select a common issue for serodiscordant partners and then use FENCE to problem solve it. The next 2 activities provided opportunities to practice the FENCE technique to discuss medication adherence, if applicable, and other decisions about their sexual relationships. Facilitators prepared couples for the next session and reintroduction into the group of couples known as the "village." As homework, couples were asked to exchange Couple Connection Coupons to request nonsexual love and affection from each other (eg, 30 minutes of cuddling).

\section{Session 5: It Takes a Village (Group Session)}

The Nguzo Saba principles of self-determination and unity were integrated into this session. While acknowledging the value of relationships, self-determination involved selfdefinition rather than being defined by others. Couples were encouraged to make their own decisions about how to stay safe and healthy, despite the perceived racism, ostracism, and stigma that they experienced in the outside world. The connection between practicing safer sex and restoring the African American community's health was highlighted by the process of Reality Construction. ${ }^{51}$ This process focused on exploring the different ways in which social injustice, fear, and perceived racism may inhibit couples from acknowledging that prevention efforts are not a method to "control behavior." Reducing risks and engaging in safer sex practices were the primary focus. The importance of knowing the history of health and educational disparities was emphasized as a way to better appreciate the resilience of African Americans who have confronted social injustice, fear, and perceived racism with strategies for social activism. ${ }^{36}$ The need for social support, a cornerstone of unity, was highlighted in this session. This information was designed to build and strengthen ethnic pride and promote individual and partner self-protection by overcoming perceived racism and stigma regarding HIV/AIDS.

Cofacilitators reintroduced the concept of the group as a village where couples can unite in a safe, respectful, and protected space and gain support. Group rules for participation, including confidentiality, and homework from the last session were reviewed.

Objectives of this session included identifying strengths of African American communities to make changes and cope with HIV/AIDS, practicing the FENCE techniques to problem-solve barriers to safer sex, and learning to identify and build social networks that promote safer sex. Each participant was asked to describe what pride in their ethnic and cultural group meant to them. Maya Angelou's poem "Still I Rise" was used to illustrate resilience and inspire couples to overcome obstacles and protect each other in the process. Each couple was asked to use the cognitive strategy FENCE to address a situation that they had experienced in which negative judgments about people with HIV interfered with a serodiscordant couple's ability to practice safer sex. The desire to avoid reminders of HIV was used as an example of how stigma promotes HIV risk practices. 
In the next activity, couples discussed the pros and cons of disclosing their HIV status to people in their support system and benefits and consequences of nondisclosure.

Single-gender groups discussed obstacles to condom use and brainstormed different strategies for working through these barriers. The mixed-gender group reconvened and shared issues discussed in the single-gender groups.

For homework, couples were encouraged to continue to work on their goals and to share Couple Connection Coupons. They were also asked to complete a worksheet to help them assess their sources of social support and to strengthen their connection to at least one person in their social network. Finally, for the Eban Closing Circle ritual, the group concluded with a Talking Circle in which each person shared what they had learned.

\section{Session 6: Strengthening the Village (Group Session)}

This session integrated principles of collective work and responsibility. Couples discussed living with HIV and participated in the Eban Fence of Safety activity, garnering support from the group or village. Couples were supported in making decisions to protect and support each other from HIV/STD transmission.

During review of the homework, couples shared their experiences with building their social network and their progress on their risk reduction goals.

The objectives of this session were to enhance identification with the group or village and to identify and problem-solve barriers to practicing safer sex. In the first activity of the session, participants were asked to take part in a group-within-a-group exercise called the Eban Fence of Safety (see Formative Study to Develop the Eban Treatment and Comparison Interventions for Couples). During this exercise, the HIV-positive partners formed an inner circle and shared how HIV affected their lives, whereas the HIV-negative partners listened as part of a protective outer circle. After discussion, the groups changed places and the HIV-negative partners shared their feelings whereas the HIV-positive partners listened. This activity was intended to help break through feelings of alienation and to enhance empathy for the challenges each partner faced.

The next activity provided several opportunities to practice the FENCE technique by problem-solving couplespecific triggers to risky behaviors. Facilitators led the group in a discussion about how (1) condoms could be incorporated into their sex life in an erotic and enjoyable way; (2) couples can be prepared to use condoms to avoid unpleasant interruptions during sexual intimacy; and (3) couples could be intimate with their partner while still protecting each other. Self-talk involving positive, motivating messages was discussed as another strategy for addressing triggers to risks and barriers to being healthy sex partners.

The couples' homework (Practice Planning Ahead for Safer Sex) included practicing the skills they learned by developing a plan to address their triggers to risky behaviors and increase safer sex. Couples were also encouraged to continue sharing the Couple Connection Coupons and to use Talk and Listen to discuss the reality of living with HIV.

\section{Session 7: Expanding the Village (Group Session)}

The primary Nguzo Saba principle underlying this session was purpose. Throughout this session, facilitators reviewed how each activity involved the shared purpose of keeping one's self, partner, family, and community safe.

For homework (Practice Planning Ahead for Safer Sex), cofacilitators asked couples to share their progress on their risk reduction and relationship goals, particularly any examples in which they utilized Talk and Listen, FENCE techniques, or other skills learned. The group offered ideas and support to couples who encountered difficulties in achieving their goals.

Objectives included reviewing skills and information, enhancing sexual communication and self-assertiveness skills, and developing strategies to stay connected to the other couples. A game called Safer Sex Jeopardy reviewed and summarized key components of the intervention: how to put on condoms correctly, HIV/STD facts, condom facts, safer sex techniques, communication and problem-solving skills, and Nguzo Saba principles. The next activity involved modeling and role-playing how to say "no" to unsafe sex in a loving way, using Talk and Listen and assertive communication. During this activity, couples learned how to consider using condoms as an intimate act of love and protection rather than symbol of mistrust or casual sex. Couples were also encouraged to use the FENCE technique to resolve situations where there might be disagreement about safer sex practices. In addition, couples were provided with Safety and Protection Cards with messages to remind themselves and each other about their commitment to safer sex and to protecting themselves, their families, and communities.

The couples' homework included creating additional personal Safety and Protection Cards to develop a message that was most meaningful to them in promoting safer sex behaviors. Because this session was the final group session, the facilitators discussed the possibility of couples exchanging contact information, if they wished to keep in touch with each other. The session ended with the Talking Circle and the Eban Closing Circle.

\section{Session 8: Celebrating our Relationship (Couple Session)}

This session reviewed all the Nguzo Saba principles but focused on self-determination, unity, and purpose to identify how to address triggers to risky behaviors, avoid relapse, and maintain safer sex behavior. Although the intervention was designed to empower couples with knowledge and skills to reduce the risk of HIV and STD transmission, the facilitators emphasized that each couple had to decide how they would use these skills to keep each other safe.

During homework review, couples shared their experiences with the Safety and Protection Cards and the Couple Connection Coupons and then reviewed progress with their couple goals. Each partner rated his or her progress on a scale from 1 to 10. The cofacilitators reviewed the couple's progress in learning the various skills taught in Eban (eg, Talk and Listen, and FENCE) and praised them for specific achievements in using skills and accomplishing their goals. 
Objectives included reinforcing safe sex communication and problem-solving skills, developing relapse prevention strategies, recommitting to staying safe, and bringing closure to the program. Couples were prepared for possible slips by identifying situations that might trigger a relapse to unsafe sexual behaviors. They were asked to role-play how to handle these potential relapse situations using the Eban skills of FENCE, Talk and Listen, and self-talk. Couples were reminded to reward themselves when they met their goal of practicing safer sex and to acknowledge any progress they achieved to build confidence in their abilities to protect themselves consistently. Examples of rewards were identified, including rewarding self-statements and engaging in safer sexual or recreational activities that both partners enjoyed. To reaffirm their commitment to practicing safer sex, participants wrote letters to themselves about the promise they made to use what they learned from the Eban Project to protect themselves and their partners from HIV and STDs. These letters were later mailed to participants to remind them of what they accomplished. The session ended with praise and recognition for the work each couple had completed and with an affirmation that focused on making healthy choices. Participants were also given Certificates of Completion and condoms.

\section{LESSONS LEARNED}

For most couples, the intervention was the first time they had ever learned skills and strategies to help improve their ability to listen and communicate with each other and make decisions together about challenging health issues. For men, in particular, it was a markedly unique experience to be in a safe and nonjudgmental environment to talk about their problems not only with their female partner but also with other African American men, who faced similar stigma, and other couples who were also in HIV-serodiscordant relationships.

The context of the intervention allowed each member of the couple to explain his or her own needs due to being infected or affected by HIV, to identify and obtain the support they needed to protect each other, and to correct misunderstandings about the challenges that each person faced. The couples reported that the Eban intervention provided an exceptional opportunity to learn about each other's experiences through both the one-on-one couple sessions and the couples group sessions. Bringing serodiscordant couples together and in groups offered the HIV-positive individuals the opportunity to talk about the challenges of living with the virus and gave the seronegative partners an opportunity to discuss their experiences and ways to cope with their relationship.

Using positive cultural values embodied in the Nguzo Saba principles, the legacy of African ancestors, and the stories of the resilience of African American slaves provided the couples with powerful examples of how they could survive the HIV epidemic. The experience of linking their history of resilience in overcoming oppression with their strengths as a community to fight the HIV/AIDS epidemic was perceived as an empowering motivational step for using condoms consistently. The principles of Nguzo Saba also helped couples reframe condom use as a symbol of love and protection as opposed to a symbol of casual sex with nonintimate partners.
Moreover, during the pilot study, couples consistently reported in their evaluations and informal feedback that their overall perception of the ethnic and cultural relevance of the Eban intervention resonated with them and enabled them to feel comfortable disclosing and working through difficult issues related to their HIV/STD risks. The significance of matching the background of the facilitators to that of the couples was another important lesson and cannot be overstated. Ethnic matching enhanced involvement and rapport and facilitated discussion of issues related to perceived racism, discrimination, and other factors that can increase risk of HIV in African American communities.

The Eban intervention's approach is unique in addressing the simultaneously occurring, complex issues that African American HIV-serodiscordant couples are likely to encounter that affect their ability to protect themselves from HIV/STDs. $\mathrm{HIV}$ is only one of many problems that these couples experienced on a daily basis, along with poverty, discrimination, oppression, health disparities, histories of trauma, childhood sexual abuse, victimization, and HIV-related stigma. Acknowledging the link between these issues and HIV risks helped to engage the couples in the intervention.

The single most helpful skill mentioned by the couples was the Talk and Listen technique. In their participant evaluations and informal feedback, couples reported that Talk and Listen enabled them to disclose sensitive issues and concerns related to HIV risks and improve their communication overall. The intervention sessions created a safe environment in which issues such as extrarelationship affairs or bisexuality were linked to HIV risks. Feedback from facilitators in the pilot study suggested that using FENCE, the cognitive strategy for problem solving, was more challenging for participants to learn than the Talk and Listen technique (see Formative Study to Develop the Eban Intervention for African American Couples). However, the participant evaluations and informal feedback from the couples did suggest that the FENCE technique provided couples with ways to find alternative solutions to condom use barriers. Couples reported that both the Talk and Listen and FENCE skills assisted them in identifying their misperceptions about each other's intentions. They became aware of irrational justifications for engaging in behaviors that heightened HIV risks.

Paying attention to couple dynamics and the relationship's context (ie, controlling behaviors, the couple's communication style, relationship dependencies, and subscription to traditional gender roles) helped them to identify how these issues affected their sexual decision making and ability to use condoms consistently.

The intervention taught technical skills on how to correctly use female and male condoms. In the beginning of the session, when the method of using condoms was taught, it was difficult for female participants to touch the model of the vagina. The women explained that viewing or touching their own body was not commonplace and, therefore, even talking about this issue caused discomfort. As a consequence, the intervention dedicated time to familiarize the couple with female anatomy before learning about condom use.

One of the concepts from Nguzo Saba is the importance of the village in promoting healthy behaviors. In the Eban 
intervention, we refer to the couples group as "our village" and the participants discussed how peer behaviors influenced their own. The Eban Project promoted the constructive use of peer norms to reinforce safer sex and reduce HIV risk. Through the "village" concept, groups of couples established a bond and developed a supportive social network among themselves. They continued this supportive connection outside the sessions.

\section{SUMMARY}

More than 2 decades into the epidemic, the elevated rates of HIV and other STDs continue to threaten African American communities. Although heterosexual intercourse remains a primary mode of transmission for both African American men and women, most HIV prevention interventions in the United States are still individual or group based and have not focused on the ongoing intimate relationships where infection often occurs. Previous research further suggests that African Americans have not responded as well as other ethnic groups to the set of evidence-based HIV prevention interventions. Consideration of culture, race, and ethnicity throughout all levels of study design and delivery of HIV prevention interventions is urgently needed.

Our experience in implementing and testing the Eban HIV risk reduction intervention leads us to the following findings. First, couples responded very well to the mixed modality of individual couple and group couple sessions to increase their motivation and ability to use condoms consistently. Second, couples were able to develop the core skills of Talk and Listen and FENCE through the course of the intervention and reported that these skills not only helped them disclose and work through their risky behaviors but also strengthened their relationship by improving their overall communication and decision-making skills. Third, a high level of cultural congruence of the intervention was achieved through matching facilitators and participants and through emphasizing Nguzo Saba principles and a perspective that takes into consideration multiple African American values, such as affirming heritage, rights and responsibilities, cultural identity, self-esteem, pride, and spiritual awareness. The Nguzo Saba principles not only served as guiding values that were woven into the content of all intervention sessions but also allowed for multiple beliefs and worldviews within this diverse community. Highlighting Nguzo Saba principles and matching facilitator-participant ethnocultural backgrounds increased the meaning and relevance of the intervention content and made participants feel comfortable disclosing and working through sensitive risk behaviors. HIV prevention studies designed for other ethnocultural couples would likely benefit from a similar process of integrating cultural values into an intervention content and delivery.

\section{REFERENCES}

1. Centers for Disease Control and Prevention. HIV/AIDS Surveillance Report 2003. Vol 15. Atlanta: US Department of Health and Human Services; CDC:2004.

2. Centers for Disease Control and Prevention. HIV/AIDS Surveillance Report. Vol 15. Atlanta: US Department of Health and Human Services; 2003.
3. Mize S, Robinson B, Bockting W, et al. Meta-analysis of the effectiveness of HIV prevention interventions for women. AIDS Care. 2002;14:163-180.

4. Kalichman SC, Kelly JA, Hunter TL, et al. Culturally-tailored HIV/AIDS risk reduction messages targeted to African American urban women: impact on risk sensitization and risk reduction. J Consult Clin Psychol. 1993;61:291-295.

5. Harris R, Bausell R, Scott D, et al. An intervention for changing high-risk HIV behaviors of African American drug-dependent women. Res Nurs Health. 1998 21:239-250.

6. DiClemente RJ, Wingood GM, Harrington KF, et al. Efficacy of an HIV prevention intervention for African American adolescent girls: a randomized controlled trial. JAMA. 2004;292:171-179.

7. Wyatt G, Myers H, Loeb T. Women, trauma, and HIV: an overview. AIDS Behav. 2004;8:401-403.

8. St Lawrence J, Wilson T, Eldridge G, et al. Community-based interventions to reduce low income, African American women's risk of sexually transmitted diseases: a randomized controlled trial of three theoretical models. Am J Commun Psychol. 2001;29:937-964.

9. Logan TK, Cole J, Leukefeld C. Women, sex, and HIV: social and contextual factors, meta-analysis of published interventions, and implications for practice and research. Psychol Bull. 2002;128:851-885.

10. Musaba E, Morrison C, Sunkutu M, et al. Long-term use of the female condom among couples at high risk of human immunodeficiency virus infection in Zambia. Sex Transm Dis. 1998;25:260-264.

11. Allen S, Tice J, Van de Perre P, et al. Effect of serotesting with counselling on condom use and seroconversion among HIV discordant couples in Africa. Br Med J. 1992;304:1605-1609.

12. Padian NS, O'Brien TR, Chang YC, et al. Prevention of heterosexual transmission of human immunodeficiency virus through couple counseling. J Acquir Immune Defic Syndr. 1993;6:1043-1048.

13. Deschamps M, Pape J, Haffner A, et al. Heterosexual activity in at risk couples for HIV infection (Abstract WC3089). Paper presented at: Seventh International AIDS Conference, Florence, Italy; 1991. Available at: http://gateway.nlm.nih.gov/MeetingAbstracts/ma?f=102193169.html.

14. Higgins DL, Galavotti C, O'Reilly KR, et al. Evidence for the effects of HIV antibody counseling and testing on risk behaviors. JAMA. 1991;266: 2419-2429.

15. Voluntary HIV-1 Counseling and Testing Efficacy Study Group. Efficacy of voluntary HIV-1 counselling and testing in individuals and couples in Kenya, Tanzania and Trinidad: a randomized trial. Lancet. 2000;356: $103-112$.

16. Glick P. Scaling up HIV voluntary counseling and testing in Africa. Eval Rev. 2005;29:331-357.

17. Allen S, Serufilira A, Bogaerts J, et al. Confidential HIV testing and condom promotion in Africa: impact on HIV and gonorrhea rates. JAMA. 1992;268:3338-3343.

18. van der Straten A, King R, Grinstead O, et al. Couple communication, sexual coercion and HIV risk reduction in Kigali, Rwanda. AIDS. 1995;9: 935-944.

19. Kamenga M, Ryder RW, Jingu M, et al. Evidence of marked sexual behavior change associated with low HIV-1 seroconversion in 149 married couples with discordant HIV-1 serostatus: experience at an HIV counselling center in Zaire. AIDS. 1991;5:61-67.

20. Weindhard L, Carey M, Johnson B, et al. Effects of HIV counseling and testing on sexual risk behavior: a meta-analytic review of published research, 1985-1997. Am J Public Health. 1999;89:1397-1405.

21. Wyatt GE, Longshore D, Chin D, et al. The efficacy of an integrated risk reduction intervention for HIV-positive women with child sexual abuse histories. AIDS Behav. 2004;8:453-462.

22. El-Bassel N, Witte SS, Gilbert L, et al. The efficacy of a relationshipbased HIV/STD prevention program for heterosexual couples. Am J Public Health. 2003;93:963-969.

23. El-Bassel N, Witte SS, Gilbert L, et al. Long-term effects of an HIV/STI sexual risk reduction intervention for heterosexual couples. AIDS Behav. 2005;9:1-13.

24. O’Leary A, Wingood GM. Interventions for sexually active heterosexual women. In: Peterson JL, DiClemente RJ, eds. Handbook of HIV Prevention. New York: Plenum Publishing Corp; 2000:179-197.

25. Kelly JA. Advances in HIV/AIDS education and prevention. Fam Relat. 1995;44:345-352.

26. Tanner WM, Pollack RH. The effect of condom use and erotic instructions on attitudes toward condoms. $J$ Sex Res. 1988;25:537-541. 
27. Ehrhardt AA. "Narrow vs broad targeting of HIV/AIDS education": response. Am J Public Health. 1994;84:498-499.

28. El-Bassel N, Witte S, Gilbert L, et al. HIV prevention for intimate couples: a relationship-based model. Fam Syst Health. 2001;19:379-395.

29. Basen-Engquist K. Psychosocial predictors of "safer sex" behaviors in young adults. AIDS Educ Prev. 1992;4:120-134.

30. Fisher JD, Fisher WA. Changing AIDS-risk behavior. Psychol Bull. 1992; 111:455-474.

31. Nadler A, Fisher JD. Volitional personal change in an interpersonal perspective. In: Klar Y, Fisher JD, Chinsky J, Nadler A, eds. Initiating Self-Change: Social Psychological and Clinical Perspectives. New York: Springer-Verlag; 1992:213-230.

32. Sormanti M, Pereira L, El-Bassel N, et al. The role of community consultants in designing an HIV prevention intervention. AIDS Educ Prev. 2001;13:311-328.

33. van der Straten A, Vernon KA, Knight KR, et al. Managing HIV among serodiscordant heterosexual couples: serostatus, stigma and sex. AIDS Care. 1998;10:533-548.

34. McKay M, Gonzales J, Stone S, et al. Multiple family therapy groups: a responsive intervention model for inner-city families. Soc Work Groups. 1995;18:7-23.

35. Bandura A. Social Foundations of Thought and Action: A Social and Cognitive Theory. Englewood Cliffs, NJ: Prentice-Hall; 1986.

36. Karenga M. Black studies and the problematic paradigm. J Black Stud. 1988;18:395-414.

37. Bronfenbrenner U. The ecology of human development. Am Psychol. 1979;32:513-531.

38. Belsky J. Child maltreatment: an ecological integration. Am Psychol. 1980;35:320-335.

39. El-Bassel N, Ivanoff A, Schilling RF, et al. Correlates of problem drinking among drug using incarcerated women. Addict Behav. 1995;20:359-369.

40. Gomez CA, VanOss Marin B. Gender, culture, and power: barriers to HIVprevention strategies for women. J Sex Res. 1996;33:355-362.

41. Amaro H, Hardy-Fanta C. Gender relations in addiction and recovery. J Psychoactive Drugs. 1995;27:325-337.

42. Ehrhardt AA, Yingling S, Zawadzki R, et al. Prevention of heterosexual transmission of HIV: barriers for women. J Psychol Human Sexual. 1992; $5: 37-67$.

43. Exner T, Gardos P, Seal D, et al. HIV sexual risk reduction interventions with heterosexual men: the forgotten group. AIDS Behav. 1999;3:347-358.
44. DiClemente RJ, Wingood GM. Expanding the scope of HIV prevention for adolescents: beyond individual level interventions. $J$ Adolesc Health. 2000;26:377-378.

45. O'Leary A, Ambrose TK, Raffaelli M, et al. Effects of an HIV risk reduction project on sexual risk behavior of low-income STD patients. AIDS Educ Prev. 1998;10:483-492.

46. Hobfoll SE, Jackson AP, Lavin J, et al. Effects and generalizability of communally oriented HIV-AIDS prevention versus general health promotion groups for single, inner-city women in urban clinics. J Consult Clin Psychol. 2002;70:950-960.

47. Misovich SJ, Fisher JD, Fisher WA. Close relationships and elevated HIV risk behavior: evidence and possible underlying psychological processes. Rev Gen Psychol. 1997;1:72-107.

48. Bandura A. Perceived self-efficacy in the exercise of control over AIDS infection. In: Mays V, Albee G, Schneider S, eds. Primary Prevention of AIDS. Newbury Park, CA: Sage; 1989:128-141.

49. O'Leary A. Self-efficacy and health. Behav Res Ther. 1985;23:437-451

50. Wyatt GE. The sociocultural relevance of sex research: challenges for the 1990s and beyond. Am Psychol. 1994;49:748-754.

51. Akoto K. Nation Building: Theory and Practice in Afrikan-Centered Education. Washington, DC: Pan-Afrikan World Institute; 1992.

52. Grills C. Motivating illegal drug use recovery: evidence for a culturally congruent intervention. J Black Psychol. 2000;26:288-301.

53. Longshore D, Grills C, Annon K, et al. Promoting recovery from drug abuse: an Africentric intervention. J Black Stud. 1998;28: 319-332.

54. Wallace BC, Constantine MG. Africentric cultural values: psychological help-seeking attitudes, and self-concealment in African American college students. J Black Psychol. 2005;31:369-385.

55. Belgrave FZ. Relational theory and cultural enhancement interventions for African American adolescent girls. Public Health Rep. 2002; 117(Suppl 1):S76-S81

56. Karenga M. Maat, The Moral Ideal in Ancient Egypt: A Study in Classical African Ethics. Los Angeles, CA: University of Southern California; 1994.

57. Triandis HC. Culture and Social Behavior. New York: McGraw Hill; 1994.

58. Markman H, Stanley S, Blumberg SL. Fighting marriage: positive steps for preventing divorce and preserving love. Contemp Psychol. 1996;41: 988-990. 